\title{
Team Resilience in Complex and Turbulent Environments: The Effect of Size and Density of Social Interactions
}

\author{
Ilaria Giannoccaro $\mathbb{D}^{1},{ }^{1}$ Giovanni F. Massari, ${ }^{1}$ and Giuseppe Carbone $\mathbb{D}^{1,2}$ \\ ${ }^{1}$ Dipartimento di Meccanica Matematica e Management, Politecnico di Bari, Viale Japigia 182, 70126 Bari, Italy \\ ${ }^{2}$ Center for Nonlinear Science, University of North Texas, P.O. Box 311427, Denton, TX 76203-1427, USA \\ Correspondence should be addressed to Giuseppe Carbone; carbone@poliba.it
}

Received 27 October 2017; Revised 20 May 2018; Accepted 12 June 2018; Published 24 July 2018

Academic Editor: Hiroki Sayama

\begin{abstract}
Copyright (c) 2018 Ilaria Giannoccaro et al. This is an open access article distributed under the Creative Commons Attribution License, which permits unrestricted use, distribution, and reproduction in any medium, provided the original work is properly cited.
\end{abstract}

\begin{abstract}
How are teams able to cope with environmental threats? Why are some teams better than others in facing this challenge? This paper addresses these questions by investigating two drivers of team resilience: the team size and the density of social interactions among team members. We adopt a complex system approach and employ a model of team decision-making where collective dynamics of team members are governed by a continuous-time Markov process. The model simulates team performance in complex and turbulent environments. It is used to measure the resilient ability of team to quickly adapt to disturbance and secure a new more desirable condition. Scenarios characterized by increasing levels of complexity and turbulence are simulated, and the resilience performance is calculated and compared. Results show that the team size negatively affects the team resilience, whilst the density of social interactions plays a positive influence, especially at a high level of complexity. We also find that both the magnitude and the frequency of disturbance moderate the relationship between team size/density and the team resilience.
\end{abstract}

\section{Introduction}

Nowadays, the complexity, the uncertainty, and the turbulence of the competitive environment continuously threat the survival of organizations. Unpredictable and large disruptive events undermining their performance are more and more frequent, from natural disasters such as hurricanes, earthquakes, seaquakes, and volcanic eruptions to terrorist attacks, human errors, and market disruptions [1-4]. Organizations not only experience sudden and occasional environmental jolts but also continuously undergo periodic shifts in demand, competitors, and regulations, which make their competitive position uncertain [5]. This situation is further complicated by the growing global interconnectedness of the environment where organizations live. Never more than now, organizations are embedded in multiple web of interactions with suppliers, customers, competitors, and institutions [6], which affect their performance in a complex, unpredictable, and nonlinear manner.

To survive and succeed in this scenario, organizations should be resilient [7]. Organizational resilience is the ability of a system to cope with perturbations, failures, and threats, by absorbing the disturbance [8-10], and/or to quickly recover, so as to restore its functions $[2,11,12]$.

The theory of organizational resilience explains how individuals, groups of individuals, and organizations as a whole are able to provide positive outcome and desirable performance under challenging and critical conditions. It includes the ability to rebounce from stressing situations, thus reducing the impact of the disruptions, but it also looks beyond entailing the ability of managing disruptions and unexpected events and maximizing the speed of recovery to the original or to a new more desirable condition $[2,13]$. Accordingly, organizational resilience involves two main dimensions: (i) resistance to change and (ii) adaptive capacity $[14,15]$. The theory of organizational resilience explains why some organizations can exist and thrive in complex environments with unpredictable, nonlinear, and nonincremental change, while others do not.

Despite the great amount of research on the topic, the theory of team resilience is still underdeveloped [16]. Team-based structures show higher resilience than hierarchy 
and bureaucratic mechanisms [4]. For example, in [17], the authors find that teams are a fundamental source of resilience in health and social care sectors.

At the team level, the theory of resilience investigates how individuals, acting collectively, enable the organization to be resilient. The majority of studies have investigated the behavioral and psychological characteristics of team members associated with high ability of the team to provide positive outcome and desirable performance, under challenging and critical conditions [18-21]. More recently, however, scholars have highlighted that grouping together resilient individuals is not a sufficient condition to make the team, as a whole, resilient. Resilience is, in fact, socially enabled and developed via the interactions and relations between the elements of the system $[7,11]$.

Thus, in order to properly investigate team resilience, it is important to focus on the interactions among individuals rather than on the individual's level of knowledge, skills, and abilities. This complex network of interactions influences both the development and the realization of resilience [15]. Team resilience spontaneously emerges from the actions of team members and their interactions. In this paper, we study the determinants of team resilience coherently with this approach, that is, focusing on the network of social interactions among the individuals in the team.

In particular, while previous studies have characterized interactions from social and psychological points of view, focusing, for example, on social capital [22] and emotional expression [23], we study the structural features of the social network resulting from the interactions among team members. We analyze the effect of size and density on team resilience, two aspects not yet investigated in the literature. Both variables influence the performance of collective decisionmaking, thereby affecting the ability of the system to adapt to disturbances. Resilience is measured in terms of efficacy of the team in performing a task when the environment is turbulent. Two dimensions are used to characterize the turbulence of the environment: (1) the magnitude of disturbance and (2) the frequency of the disturbance. The magnitude corresponds to the extent to which the event is critical for the team performance. The frequency corresponds to the dynamicity of the change. In particular, we investigate how and whether size and density influence team resilience and study the moderating role played by the magnitude and the frequency of disturbance on this relationship.

To accomplish our research aim, we use simulation as research methodology and adopt the model developed first by Carbone and Giannoccaro [24] and then by De Vincenzo et al. [25], which reproduces how individuals collectively make decisions in complex but static environments. Several agent models can be found in the literature aimed at reproducing the decision-making process in groups [19-21, 26, 27]. In our model, the team is framed as a complex system made up of agents (individuals) making decisions and their social interactions. Individuals make decisions pushed by two drivers: (1) the improvement of a fitness level by measuring, on the basis of members' knowledge, how good the decisions are for the organization and (2) the seeking of consensus with the other interacting members.
The fitness levels associated with the decisions are generated following the classical $N K$ fitness landscape procedure [28-31], where $N$ corresponds to number of decisions and $K$ to the interdependence among the decisions. $N$ and $K$ control the complexity of the environment. Consensus seeking is modelled by using the Ising-Glauber dynamics [32].

This research methodology is chosen for several reasons. It is consistent with a long tradition of creating simple yet insightful models of the organizations as complex adaptive systems, by means of $N K$ fitness landscape in both singlefirm [31, 33-36] and multifirm [37-42] contexts. In these stream of studies, the organization is supposed to search for high-performing combinations of $N$ interdependent decisions (choice configurations) [43]. Therefore, the organization is solving a decision-making problem interpreted as a performance landscape. In particular, the $N K$ fitness landscape consists in the map of all the choice configurations onto the attendant performance. The organization undertakes an adaptive walk on the fitness landscape to discover the highest peak. The merit of $N K$ fitness landscape is allowing the modeler to control and fine-tune the environmental complexity and turbulence in an easy manner [33].

According with this approach, in our model, we study the team as a complex adaptive system made up of individuals collectively searching on the performance landscape. Since the environment is turbulent and disturbances occur, the team, as a whole, adapts to change and reacts to the disturbance by choosing new combinations of decisions in order to reach the same or a better performance than those before the disruption. For this reason, our model belongs to the class of models studying resilience according to a dynamics perspective.

A further merit of our approach is that team resilience emerges from the bottom as the spontaneous result of individuals' actions and their interactions. It is a collective property and not simply the result of the existence of resilient individuals forming the team. This permits to shed light on the relationship between individual actions and organizational resilience, being this a crucial point to be further investigated and clarified [15]. To model the influence of social relationships, we employ the Ising-Glauber dynamics [32]. The Ising methodology has been successfully employed in social science, economics, and management science, to model the complex dynamics of opinion formation inside groups, by also considering that each individual opinion is affected by the opinion of his/her neighbors [24, 44-48]. The reason for employing the Ising-Glauber dynamics in a team context is justified by social influence theory. This theory argues that individuals make changes to their feelings, behaviors, and decisions, as a result of the interaction with the others [44]. Therefore, this model applies very well to teams, where individual's decision is affected by the opinion of neighbors or interacting members [24, 25, 44-46, 49-51].

In this paper, we simulate the team dynamics in environments characterized by increasing complexity and increasing levels of disturbance both in magnitude and frequency. We then compute resilience performance. Finally, a simulation analysis is carried out to investigate the influence of team size and density of interactions on team resilience. Multiple 
scenarios are considered, consisting of teams with different sizes and densities of social interactions.

Our study shows that the team size negatively affects the team resilience. Furthermore, both the magnitude and the frequency of disturbance negatively moderate the relationship between the team size and the team resilience. As to the density, we find that the density of social interactions positively influences the team resilience, while the magnitude of disturbance (frequency) negatively (positively) moderates the relationship between the density and the team resilience.

The paper is organized as follows. We first provide the theoretical background of the study, by underlying the definitions of organizational resilience and carrying out a brief review of its main drivers. Then, we describe the model of team collective decision-making in terms of dynamics, model of environment, and performance. Successively, we discuss the simulation analysis carried out using the model, and the results are achieved. We end with conclusions concerning limitations and further research.

\section{Organizational Resilience}

2.1. Definitions. Resilience is both a multifaceted and multidimensional concept [52]. The term originated in ecology and environment field of studies and was introduced by Holling [8], who defined the resilience of an ecosystem as its ability to absorb change and still exists. Resilience is related to stability and the ability of the system to return to the original equilibrium after perturbation.

Since Holling's seminal contribution, the concept has attracted the attention of scholars in multiple and even distant fields such as physics, engineering, psychology, computer science, economics, and management. It has been applied to multidisciplinary contexts, such as energy supply networks, supply chains, organizational teams, computer networks, transportation, and financial systems.

Despite this multidisciplinary nature, scholars agree with the definition of resilience recognizing two main perspectives, that is, the static and dynamic ones $[13,14,53]$. The resilience is static when it focuses on the ability of the system to absorb disturbance and bounce back to the original equilibrium state, maintaining its core functions when shocked $[6,9]$. In such a case, the resilience is tied to the property of hardiness and robustness of materials, as the ability to recover the original shape and features once stretched. The dynamic perspective focuses on the ability of the system to evolve over time moving towards the original but even new, more favorable equilibrium state $[2,54,55]$. Rather than focusing on the ability of the system to resist to change, this perspective puts the attention on the adaptive capacity of the system, which is able to react to disturbance by changing its structure, processes, and functions in order to increase its ability to persist [56]. In this case, the system is resilient not because it is able to absorb disturbance, preserve organizational functioning, and recover but because it adapts to change.

These two opposite perspectives of resilience are important especially for organizational systems, because different organizational features are required to provide them. The ability to resist to change needs to develop a "defence" approach to the threat [16]. In such a case, organizations should provide themselves of monitoring capability to detect and anticipate the critical event and should consider to increase diversity and redundancy of resources [57].

The adaptive capacity needs an "offence" proactive approach. In such a case, the disturbance is seen as an opportunity not a threat and resilience requires the ability to activate, combine, and extend the resources as the adverse conditions arise [2]. Resilient organizations activate positive feedback loops that refine and strengthen their capabilities in order to avoid rigidity and create opportunities from the adverse situations [15].

Finally, even though in the majority of studies the organizational resilience is viewed as a positive characteristic, a recent contribution [16] recognizes that it can be desirable or undesirable, depending on the system state. When the system operates at not desirable conditions for key stakeholders, resilience is a negative property because it pushes the system to resist change and restore a negative state.

2.2. Drivers of Organizational Resilience. There is a limited literature that explores the drivers of organizational resilience [4]. The psychological school notes that the resilience of organization "builds on the foundation of the resilience of members of that organization" [12]. Individual resilience is defined as "the capacity to rebound or bounce back from adversity, conflict, failure, or even positive events, progress, and increased responsibility" [58]. It depends on behavioral and psychological traits of individual. Werner and Smith [59] find that resilient individuals are characterized by problem solving abilities, favorable perceptions, positive reinforcement, and strong faith. Masten [60] identifies cognitive abilities, temperament, positive self-perceptions, faith, a positive outlook on life, emotional stability, self-regulation, a sense of humor, and general appeal or attractiveness as potential assets that can contribute to higher resiliency. Fisk and Dionisi [61] investigate self-monitoring, self-efficacy, self-evaluation, the five personality traits, and emotional intelligence. Coutu [62] finds that common features of resilient individuals are the acceptance of reality, the belief that life is beautiful, and ability to improvise.

At the organizational level, resilience should be seen as much as an individual trait [63]. Organizational resilience is rooted in the complexity "logic." It is more than the additive combination of individual capabilities [64]. It is a collective and emerging capacity of the system resulting from both the actions of individuals and their interactions [15]. For example, assembling a group of resilient individuals does not assure to obtain a resilient team. The lack of communications, the existence of conflicts concerning leaderships and decisions, and the lack of a social identity can undermine the ability to react and quickly adapt to a changing environment [65].

In this respect, the network of complex interactions among individuals is critical to enhance resilience. Good relations and constructive interactions are recognized as key features to enhance team resilience [58]. Stephens 
et al. [23] find that the quality of emotional expression characterizing interactions is a source of team resilience. Morgan et al. [18] show four main resilient characteristics of elite sport teams: group structure, mastery approaches, social capital, and collective efficacy.

Organizational resilience is also related to the organizational structure. Decentralized decision-making structures, network approaches, and team-based organizations show higher adaptive capacity than hierarchy and centralized authority $[4,66,67]$.

\section{The Model}

We consider a team of $M$ individuals collectively performing a task in a complex and turbulent environment. The team decision-making process is modelled referring to the model first developed by Carbone and Giannoccaro [24] and then by De Vincenzo et al. [25]. Here, the team is engaged in solving a combinatorial decision-making problem, consisting in identifying the combination of multiple and interdependent decisions, yielding to the highest payoff for the organization. For example, consider a sourcing team which is assigned with the task to procure raw materials for a company. This task can be conceptualized in terms of interdependent decisions, such as looking for potential suppliers, choosing the supplier, and preparing the contract. The team should make the decisions so as assuring a high performance to the company. Each specific combination of choices is assigned with a fitness value, measuring how good is that combination for the company, using the $N K$ fitness landscape, where $N$ stands for multiple decisions (assumed to be binary) and $K$ for the interdependence among them. This problem space (referred as fitness landscape) consists therefore of $2^{N}$ possible combinations of choices on activities. Specifically, the $N K$ fitness landscape is generated by following a stochastic procedure, which permits to assign the payoff, $P(\mathbf{d})$, to each combination of choices on decisions $\mathbf{d}=\left(d_{1}, d_{2}, \ldots\right.$, $\left.d_{N}\right)$. The payoff value, $P(\mathbf{d})$, is computed by the following formula [25]:

$$
P(\mathbf{d})=\bar{V}+\sqrt{N}[V(\mathbf{d})-\bar{V}]
$$

where

$$
V(\mathbf{d})=\frac{\sum_{j=1}^{N} C_{j}(d)}{N}
$$

and $\bar{V}$ is the statistical average of $V(\mathbf{d}) . C_{j}$ is the contribution that the decision $j$ leads to the total system payoff. The latter is drawn at random for a uniform distribution $[0,1]$. Notice that, as effect of the interdependencies among decisions, $C_{j}$ depends not only on how the decision $j$ is resolved but also on the interdependent decisions. $K$ controls the complexity of the landscape. The higher $K$, the more complex the landscape is. Solving a NK Kaufmann combinatorial problem, that is, finding the optimum of the $N K$ landscape, is classified for $K>2$ as a NP- complete problem [68] (for details about the landscape generation see $[25,28,29,37-41,69])$.

3.1. Team Collective Decision-Making Process. We consider that any individual $k$ formulates his/her own opinion $\boldsymbol{\sigma}_{k}=($ $\left.\sigma_{k}^{1}, \sigma_{k}^{2}, \ldots, \sigma_{k}^{N}\right)$ concerning the preferred combination of choices on decisions, so as to optimize a personal payoff function (perceived payoff), which depends on the level of knowledge of the individual about the problem. The latter is coded by means of the probability $p$ that each single agent knows the contribution $C_{j}(\boldsymbol{\sigma})$ to the total fitness. Being $\mathbf{D}$ the matrix whose element $D_{k j}$ takes the value of 1 with probability $p$ and 0 with probability $(1-p)$. The perceived fitness of the agent $k$ is so defined:

$$
V_{k}\left(\boldsymbol{\sigma}_{k}\right)=\frac{\sum_{j=1}^{N} D_{k j} C_{j}\left(\boldsymbol{\sigma}_{k}\right)}{\sum_{j=1}^{N} D_{k j}}
$$

However, we also consider that the natural tendency of individuals to avoid conflicts and be in agreement with the people they interact with pushes them to modify their own opinions, taking into account the opinions of the other team members [70].

The dynamic process is modelled by means of a continuous-time Markov process, whose transition rates are defined to capture these two drivers of individual behavior in teams $[24,25]$.

We consider that each agent $k$ is characterized by the state vector $\boldsymbol{\sigma}_{k}=\left(\sigma_{k}^{1}, \sigma_{k}^{2}, \ldots, \sigma_{k}^{N}\right)$ with $k=1,2, \ldots, M \cdot \sigma_{k}^{j}$ with $j=1,2, \ldots, N$ is a binary variable modeling the opinion of the individual $k$ on the decision $d_{j}$. It is a binary variable $(-1,+1)$.

Each individual interacts with the other team members expressing its own opinion on each decision and listening to the opinion of the other team members.

We describe the social interactions occurring among the individuals by means of a multiplex network, where each layer corresponds to the specific decision $d_{j}$. On each layer, the nodes are the individuals and the links are the social interactions occurring among the team members and concerning that specific decision. The multiplex network is described by a $N$-block adjacency matrix A (see Figure 1).

The state of the whole system is then defined by the vector $\mathbf{s}=\left(s_{1}, s_{2}, \ldots, s_{l}, \ldots, s_{M \times N}\right)=\left(\sigma_{1}^{1}, \sigma_{1}^{2}, \ldots \sigma_{1}^{N}, \sigma_{2}^{1}, \sigma_{2}^{2}, \ldots \sigma_{2}^{N}\right.$ $\left., \ldots, \sigma_{M}^{1}, \sigma_{M}^{2}, \ldots \sigma_{M}^{N}\right)$. The dynamics of the system opinions (spins) is governed by means of a continuous-time Markov chain where the probability $P(\mathbf{s}, t)$, which at time $t$, the state vector takes the value $\mathbf{s}$ out of $2^{M \times N}$ possible states, satisfies the following master equation:

$$
\frac{d P(\mathbf{s}, t)}{d t}=-\sum_{l} w\left(\mathbf{s}_{l} \rightarrow \mathbf{s}_{l}^{\prime}\right) P\left(\mathbf{s}_{l}, t\right)+\sum_{l} w\left(\mathbf{s}_{l}^{\prime} \rightarrow \mathbf{s}_{l}\right) P\left(\mathbf{s}_{l}^{\prime}, t\right)
$$

where $\mathbf{s}_{l}=\left(s_{1}, s_{2}, \ldots, s_{l}, \ldots, s_{M \times N}\right)$ and $\mathbf{s}_{l}^{\prime}=\left(s_{1}, s_{2}, \ldots,-s_{l}\right.$, $\left.\ldots, s_{M \times N}\right)$. 

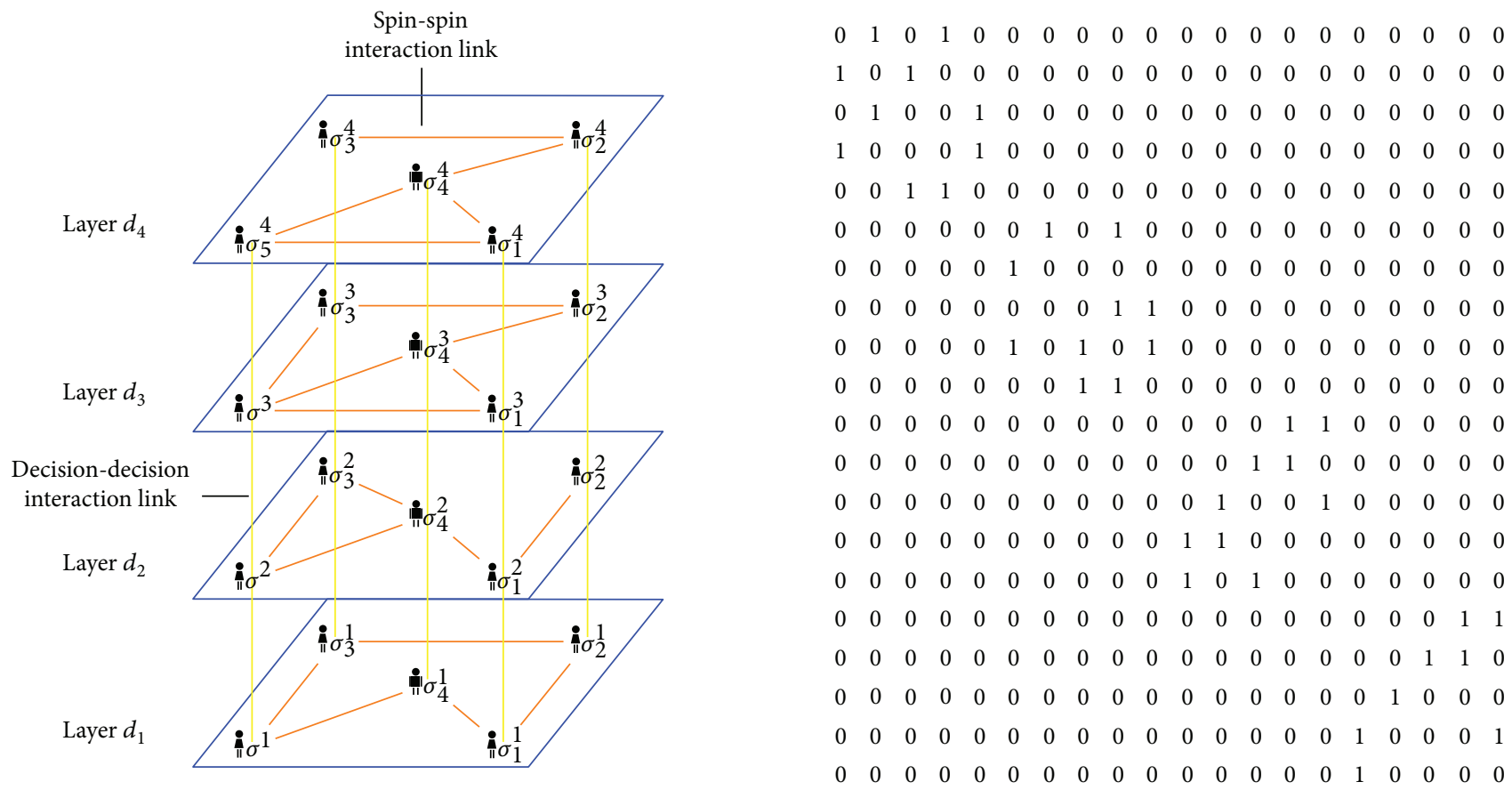

Figure 1: The multiplex network describing team social structure.

The transition rate of the Markov chain (i.e., the probability per unit time that the opinion $s_{l}$ flips to $-s_{l}$ while the others remain temporarily fixed) is so defined:

$$
w\left(\mathbf{s}_{l} \rightarrow \mathbf{s}_{l}^{\prime}\right)=\frac{1}{2}\left[1-s_{l} \tanh \left(\frac{\beta J}{\langle\kappa\rangle} \sum_{k} A_{l k} s_{k}\right)\right] \exp \left\{\beta^{\prime}\left[\Delta V\left(s_{l}^{\prime}, s_{l}\right)\right]\right\}
$$

The transition rate is the product of two terms: (1) an Ising-like term based on the Glauber dynamics [32] that models the process of consensus seeking aimed at minimizing the level of social conflict and (2) the Weidlich exponential rate [71], which models the optimizing behavior of the agents [25]. In particular, the Ising-like term pushes the individual to flip the opinion $s_{l}$ depending on the level of disagreement with the other team members [32]. The higher the level of disagreement, the higher the probability to change the opinion. Similarly, the Weidlich rate pushes the individual to flip the opinion $s_{l}$ depending on the improvement in the individual perceived fitness that the flip would determine.

Note that $A_{l k}$ is the element of the $N$-block adjacency matrix A. $\beta J$ models the social interaction strength measured in units of temperature $\beta^{-1}$. $\langle\kappa\rangle$ is the mean degree of the network of interactions among the agents on each decision layer. $\beta$ corresponds to the level of trust the members have in the judgement or opinion of others. Similarly, the $\beta^{\prime}$ is related to the level of confidence the members have about their perceived fitness.
We employ the Gillespie algorithm [72, 73], a wellestablished simulation algorithm able to exactly solve a Markov time-continuous chain as the one reported in (4). Thus, at each iteration, the time step of the next opinion change and the opinion change itself are computed. Since the time steps follow an exponential distribution, whose parameters depend on the transition rate and the size of the team, the simulation time length is, in average, shorter or longer depending on the speed of diffusion of opinions within the network. This is taken into account when team performance are computed (see Section 3.2).

3.2. Group Performance. At each time step, the team should select a combination of choices on decisions $\mathbf{d}(t)$, given the state vector $\mathbf{s}(t)$. To do this, we adopt the majority rule, that is, the team choice on each decision is the one chosen by the majority of the team members. We select the majority rule because it is consistent with our theory concerning consensus reaching inside the group. The individuals tend to make decisions to reduce the disagreement, but this does not 
necessarily imply that they will make the same decisions and at the end, they will totally agree on a given configuration. Therefore, since individuals in the team make their personal combination of decisions, we use a realistic rule, that is, the majority rule, on each single decision, to identify the team configuration at each iteration step. Moreover, the majority rule is proven to perform better than best and random member rules in different situations $[74,75]$.

Given the set of opinions $\left(\sigma_{1}^{j}, \sigma_{2}^{j}, \ldots, \sigma_{M}^{j}\right)$ that the agents have about the decision $j$ at time step $t$, we set the team's choice on the decision $j$ as follows:

$$
d_{j}(t)=\operatorname{sgn}\left[M^{-1} \sum_{k} \sigma_{k}^{j}(t)\right], \quad j=1,2, \ldots, N .
$$

If $M$ is even and in the case of a parity condition, $d_{j}$ is uniformly chosen at random between the two possible values $+1,-1$.

At each time step, the team fitness performance is then calculated as $V(t)=V[\mathbf{d}(t)]$. This quantity measures the efficacy of the solution found by the team at the time step $t$. For additional details about the model, the reader is referred to $[24,25]$.

3.3. Turbulent Environment. Recalling that the time interval between two consecutive steps of the Gillespie algorithm (see the Appendix) is large or short depending on whether the speed of opinion diffusion within the network is correspondingly slow or fast, we use the simulation step, instead of time, to represent the results of our simulations. This is equivalent to scaled time accordingly to the speed of diffusion. This specific choice comes from the fact that the dependence of resilience on the speed of diffusion is too evident; a low speed of diffusion hampers the ability of the system to change its configuration and adapt to a new environment. Therefore, considering that larger teams are necessarily characterized by a lower speed of opinion diffusion, we preferred to represent the results in terms of number of steps with the aim of isolating the effect of structural properties on resilience, besides their influence on the diffusion time. If no disturbance or event occurs during the simulation period, the environment is static. To model turbulence in the environment, we introduce disturbance during the simulation period. In particular, the disturbance corresponds to a critical event that modifies the $N K$ fitness landscape [33].

Two dimensions are used to characterize the turbulence of the environment: (1) the magnitude and (2) the frequency of the disturbance. The magnitude corresponds to the extent to which the critical event modifies the payoffs associated with actions. We model this by means of the level of correlation between the landscapes. A high correlation means that the configurations will tend to maintain the same payoffs before and after the disturbance and vice versa. To this end, at each critical event, we define the new quantities $C_{j}$ as

$$
C_{j}=\bar{V}+\frac{1}{\sqrt{1+\zeta^{2}}}\left[\left(C_{0 j}-\bar{V}\right)+\varepsilon\right],
$$

where $C_{0 j}$ is the undisturbed contribution to the fitness landscape and $\varepsilon$ is a normal distributed random noise, with zero average. The quantity $\zeta=\sigma_{\varepsilon} / \sigma_{C_{0 J}}$ is the ratio between the standard deviations of $\varepsilon$ and $C_{0 j}$, respectively. This choice guarantees that the standard deviation $\sigma_{C_{j}}$ of the new stochastic quantity $C_{j}$ satisfies the relation $\sigma_{C_{j}}=\sigma_{C_{0 j}}$. Moreover, the correlation coefficient $\tau$ between the newly generated landscape $V$ and the old one $V_{0}$ is given by

$$
\tau=\frac{\left\langle V V_{0}\right\rangle}{\sqrt{\left\langle V^{2}\right\rangle\left\langle V_{0}^{2}\right\rangle}}=\frac{1}{\sqrt{1+\zeta^{2}}} .
$$

The frequency of disturbance considers how fast the environment is changing. A high frequency corresponds to environments fast moving, irrespective of the magnitude of change. We model this by means of the parameter $(\Delta)$, that is, the number of times the critical event (i.e., the change of the landscape) occurs over the given simulation period. Note that the frequency of disturbance is defined with respect to the total number of iteration steps, that is, referring to the scaled time. Therefore, each disturbance, that is, each change of the landscape, occurs after a given number of iteration steps, depending on the frequency chosen.

3.4. Team Resilience Measurements. We measure team resilience by capturing the ability of the team to adapt to disturbance and identify a new desirable condition characterized by high fitness. This is consistent with the definition of resilience as the ability of the team to provide positive outcome and desirable performance, under challenging and critical conditions [18].

In particular, we compute the resilience performance of the team by averaging the team fitness performance $V(\mathbf{d})$ at each simulation step (in percentage of the maximum payoff achievable on the landscape) across all the simulation steps. The higher this value, the higher team resilience is. This measure of resilience is consistent with other works on the topic $[33,38]$.

\section{Simulation Analysis}

We consider a team of size $M$ engaged in solving a combinatorial decision-making problem characterized by $N=12$. We set $\beta^{\prime}=10, \beta J=0.5$, and $p=1$. Following the analysis presented in [25], the values of the strength of social interaction $\beta J$ and the self-confidence $\beta^{\prime}$ have been chosen such that, in the case of no disturbances (baseline model), the size of the team does not affect its performance. Twelve environmental scenarios are simulated resulting from the combination of three values of complexity $(K=1,3,11)$, two values of magnitude of disturbance $(\zeta=0.75,4.89)$ corresponding to correlation coefficient values $\tau=0.2,0.8$, respectively, and two values of frequency of disturbance $(\Delta=10,20)$. To analyze the effect of team size and density, we perform simulations changing the value of $M$ (5 and 11$)$ and the density $(\sim 0.33$, $\sim 0.67,1)$. In the case of density $<1$, a random pattern is set for the social network of the interactions. Each scenario is replicated 300 times for a simulation period of 100.000 iteration steps. 
TABLE 1: Results for $M=5$ and $M=11$ with density $=1 .^{*}$

\begin{tabular}{|c|c|c|c|c|c|c|c|}
\hline & & \multicolumn{3}{|c|}{$\zeta=0.75$} & \multicolumn{3}{|c|}{$\zeta=4.89$} \\
\hline & & $M=5$ & $M=11$ & Difference \% & $M=5$ & $M=11$ & Difference \% \\
\hline \multirow{4}{*}{$K=1$} & $\Delta=10$ & 0.98508 & 0.98980 & $0.48 \%$ & 0.88537 & 0.79116 & $-10.64 \%$ \\
\hline & Std. dev. & 0.02049 & 0.01271 & & 0.11081 & 0.11164 & \\
\hline & $\Delta=20$ & 0.98827 & 0.99306 & $0.48 \%$ & 0.88319 & 0.79320 & $-10.19 \%$ \\
\hline & Std. dev. & 0.01953 & 0.01103 & & 0.09567 & 0.08294 & \\
\hline \multirow{4}{*}{$K=3$} & $\Delta=10$ & 0.97759 & 0.97829 & $0.07 \%$ & 0.88434 & 0.80238 & $-9.27 \%$ \\
\hline & Std. dev. & 0.03352 & 0.02772 & & 0.11891 & 0.11421 & \\
\hline & $\Delta=20$ & 0.97868 & 0.97616 & $-0.26 \%$ & 0.86897 & 0.79684 & $-8.30 \%$ \\
\hline & Std. dev. & 0.02900 & 0.03364 & & 0.13352 & 0.12690 & \\
\hline \multirow{4}{*}{$K=11$} & $\Delta=10$ & 0.82976 & 0.76812 & $-7.43 \%$ & 0.74432 & 0.59060 & $-20.65 \%$ \\
\hline & Std. dev. & 0.23705 & 0.26384 & & 0.27678 & 0.30948 & \\
\hline & $\Delta=20$ & 0.75272 & 0.66394 & $-11.79 \%$ & 0.65326 & 0.49117 & $-24.81 \%$ \\
\hline & Std. dev. & 0.26848 & 0.28888 & & 0.29882 & 0.29246 & \\
\hline Mean & & 0.91868 & 0.89489 & $-3.07 \%$ & 0.81991 & 0.71089 & $-13.98 \%$ \\
\hline
\end{tabular}

${ }^{*}$ Differences in results are significant with $p<0.001$ ( $t$-test), except for the cases with $K=1$ and $\zeta=0.75$ and the case with $K=3$ and $\Delta=10$ and 0.75 .

The resilience performance is computed by averaging the results in terms of efficacy $V / V_{\max }$ across iteration steps and replications.

\section{Results}

5.1. The Effect of Size. Table 1 summarizes the results achieved by the teams characterized by $M=5$ and $M=11$ and density $=1$ for all the twelve environmental scenarios.

As complexity rises $(K=1,3,11)$, performance decreases, regardless of the values of magnitude and frequency of disturbance. For example, in the case of $\Delta=20$ and $M=5$, the performance is $0.98508,0.97759$, and 0.82976 for $K=1,3$, 11 , respectively. We also note that in all cases the team performance diminishes as the magnitude of the disturbance increases (moving from $\zeta=0.75$ to $\zeta=4.89$ ). For example, for $\Delta=20$ and $M=5$, the performance decreases from 0.98508 to 0.88537 as the magnitude rises. These trends run as expected, confirming the validity of our simulation model.

We now analyze the direct effect of team size on the team resilience. To do this, we compute the performance difference between the cases with $M=5$ and $M=11$ in percentage to $M=5$ (see difference $\%$ in Table 1 ) for any $K$, fixed $\zeta$. Except for few cases where differences are not significant (for $K=1, \Delta=10,20$ and $\zeta=0.75$ and for $K=3, \Delta=10$ and $\zeta=0.75)$, in all the other cases, the performance difference is significant and negative. This means that team size negatively affects team resilience. Larger teams are less able to adapt to disturbance and recover to configurations with high performance. This follows from the fact that in large teams the consensus seeking is slower than in small teams and achieving a highly agreed solution is more difficult.

We also investigate the moderating effect of the magnitude of disturbance on the relationship between team size and the team resilience. We compare the performance difference in the case of low versus high magnitude of disturbance. We achieve that on average the performance decrease is higher for $\zeta=4.89$. In particular, this difference on average is equal to $-3.07 \%$ and $-13.98 \%$ in the case of $\zeta=0.75$ and $\zeta=4.89$, respectively. This means that the magnitude of disturbance plays a negative moderating effect on the relationship between team size and team resilience. In the case of disturbances with high impact on the environment, the negative effect of large-sized teams on resilience is more pronounced.

In fact, when the level of disturbance is high (i.e., when the landscape is changing quite a lot), team needs to make new and strongly different decisions in order to reach an optimal solution. However, large teams need more time to agree on new decisions than small teams, thereby showing worse performance compared to the latter.

We finally investigate the moderating effect of the frequency of disturbance on the relationship between the team size and resilience. First, we note that, except for the cases with $K=1$ whose performance difference is not significant, the frequency of disturbance negatively affects resilience. For example, in the case of $M=5$ for $K=3$ and $\zeta=4.89$, the performance decreases from 0.88434 to 0.86897 moving from $\Delta=10$ to $\Delta=20$. Moreover, it is noteworthy that, as the team size rises from $M=5$ to $M=11$, the decrement in the performance becomes more important as the frequency of disturbance rises. In particular, on average such a performance decrease due to size growth is $-7.91 \%$ and $-9.15 \%$ for $\Delta=10$ and $\Delta=20$, respectively. This means that the frequency of disturbance negatively moderates the relationship between the team size and team resilience. In environments characterized by high dynamicity, the negative effect of team size is more pronounced. Since, as said above, a large number of individuals reach consensus on a good solution in longer time, compared to a small number of individuals, fast changing environments strongly prevent large teams from finding good solutions, making them exhibit low performance.

5.2. The Effect of Density. In Table 2, the results concerning teams with $M=11$ and three increasing values 
TABLE 2: Results for $M=11$ with density $=1 .^{*}$

\begin{tabular}{|c|c|c|c|c|c|c|c|c|c|}
\hline & \multicolumn{4}{|c|}{$\zeta=0.75$} & \multicolumn{4}{|c|}{$\zeta=4.89$} \\
\hline & & $d=0.33$ & $d=0.67$ & $d=1.0$ & Diff. \% & $d=0.33$ & $d=0.67$ & $d=1.0$ & Diff. \% \\
\hline \multirow{4}{*}{$K=1$} & $\Delta=10$ & 0.989 & 0.990 & 0.990 & 0.001 & 0.789 & 0.792 & 0.791 & 0.002 \\
\hline & Std. dev. & 0.014 & 0.013 & 0.013 & & 0.125 & 0.113 & 0.112 & \\
\hline & $\Delta=20$ & 0.992 & 0.993 & 0.993 & 0.001 & 0.765 & 0.775 & 0.789 & 0.031 \\
\hline & Std. dev. & 0.012 & 0.011 & 0.011 & & 0.093 & 0.083 & 0.083 & \\
\hline \multirow{4}{*}{$K=3$} & $\Delta=10$ & 0.969 & 0.977 & 0.978 & 0.009 & 0.772 & 0.804 & 0.802 & 0.039 \\
\hline & Std. dev. & 0.042 & 0.030 & 0.028 & & 0.143 & 0.117 & 0.114 & \\
\hline & $\Delta=20$ & 0.969 & 0.976 & 0.976 & 0.007 & 0.759 & 0.794 & 0.797 & 0.050 \\
\hline & Std. dev. & 0.035 & 0.024 & 0.023 & & 0.157 & 0.126 & 0.127 & \\
\hline \multirow{4}{*}{$K=11$} & $\Delta=10$ & 0.713 & 0.753 & 0.768 & 0.077 & 0.563 & 0.581 & 0.591 & 0.049 \\
\hline & Std. dev. & 0.297 & 0.275 & 0.264 & & 0.307 & 0.308 & 0.309 & \\
\hline & $\Delta=20$ & 0.653 & 0.665 & 0.664 & 0.017 & 0.498 & 0.489 & 0.491 & -0.014 \\
\hline & Std. dev. & 0.303 & 0.290 & 0.289 & & 0.295 & 0.294 & 0.292 & \\
\hline Mean & & 0.881 & 0.892 & 0.895 & 0.019 & 0.691 & 0.706 & 0.710 & 0.026 \\
\hline
\end{tabular}

* Standard deviation is reported below. Differences in results are significant with $p<0.001$ ( $t$-test), except between the cases for $K=1$ and $\zeta=0.75$.

of density are shown for all the twelve environmental scenarios considered.

We compare the performance as the density increases, fixed $K, \zeta$, and $\Delta$. In particular, we computed performance difference between the cases with $d=0.33$ and $d=1.0$ in percentage to $d=0.33$ (see diff. \% in Table 2). Results show that the density does not affect performance when the level of complexity is low $(K=1)$, regardless of the frequency and the magnitude of disturbance. For higher levels of complexity $(K=3,11)$, the density positively influences team performance, even though the performance increase is quite small. Higher densities in fact tend to increase the exchange of information among the members, thus making the exploration of the new environment more effective.

By comparing the performance increase in the case of low versus high magnitude of disturbance, we note that on average it is higher when the magnitude is high. In particular, the performance increase is on average 0.019 and 0.026 for $\zeta=$ 0.75 and $\zeta=4.89$, respectively. This shows that the magnitude of disturbance plays a positive moderating effect on the relationship between density and team resilience. In environments characterized by disturbance with high impact, the positive effect of density of social interactions on resilience is more pronounced. In fact, a larger number of connection improves the information flow and sharing among the individuals, thus making the team more rapid in reaching consensus on good solution and able to tolerate high disturbances and to reach higher performance in strongly noisy (disturbed) environments.

We finally analyze the moderating effect of frequency on disturbance on the relationship between density and team resilience. To do this, we compare the performance difference between $d=0.33$ and $d=0.1$ achieved in the cases of $\Delta=10$ and $\Delta=20$. On average, this performance difference tends to decrease with the frequency of disturbance. In particular, it is equal on average to $1.54 \%$ and $2.96 \%$ for $\Delta=10$ and $\Delta=20$, respectively. This means that the frequency of disturbance negatively moderates the relationship between density and the team resilience. In environments characterized by high dynamicity, the positive effect of density on resilience is less pronounced. In fact, if the frequency of the disturbance is too high, there is no enough time for the individuals to find an agreement and make good decision. Thus, the beneficial effect coming from more connections which permit the system to share information is reduced.

\section{Discussion and Conclusions}

This paper investigated team resilience in complex and turbulent environments. In particular, we employed a simulation model reproducing team collective behavior in solving combinatorial task, to measure the ability of the team to adapt to the change and secure desirable performance. Our model is consistent with the complex system approach to study resilience, being the team framed as a network of agents (individuals) making decisions, connected by means of social interactions. In doing so, resilience is an emergent property of the team.

Our analysis focused on two drivers of team resilience, which have not yet been investigated in the literature: the team size and the density of the network of social interactions. Both aspects refer to network properties of the team and not on individual features. This responds to the call for considering resilience as a socially enabled property and for studying those determinants concerning the coordination of the social interactions among team members rather than the behavioral and psychological characteristics of team members.

We found that both team size and density of social interactions, which influence the collective performance of the individuals to solve complex problems, affect team resilience. In particular, we achieved that the team size negatively affects team resilience, while the effect of density is beneficial for resilience, even though considerably lower and significant only for high levels of complexity of the environment. 
We also found that both the magnitude and the frequency of disturbance moderate the relationship between the team size/density and team resilience. In particular, we showed that the magnitude and frequency of the disturbance negatively moderate the relationship between team size and team resilience, while the magnitude (frequency) positively (negatively) moderates the relationship between density and team resilience.

These findings contribute to the literature suggesting how to design teams more resilient to environmental turbulence. In particular, based on them, we suggest preferring teams with small size especially when the environment is characterized by high-impact critical events occurring with high frequency. Even though the influence of density is less significant, we suggest to favor the social interactions among team members to improve team resilience, especially when the environment is characterized by high complexity and the critical event has high impact on performance.

Our study suffers some limitations. These findings are achieved under specific values of $\beta, \beta^{\prime}$, level of self-confidence, and the strength of social relationships, respectively. As shown in [25], different values of these parameters can modify the ability of teams to perform well and consequently its resilience.

Further research will be devoted to analyze further determinants influencing the coordination of social interactions. In particular, it could be interesting to analyze the effect of different network structures (e.g., smallworld and scale-free), the presence of distrust relationships among some of the individuals, the influence of the differences among the individuals in terms of propensity to the opinion change, or rather to investigate the effect of team leaders on resilience.

\section{Appendix \\ The Gillespie Algorithm}

The stochastic simulation algorithm we used to solve the Markov process (5) is derived from the one proposed by Gillespie [72, 73]. We just summarize the main steps of the algorithm:

(1) Choose at random the initial state $\sigma$ of the system.

(2) Calculate all the transition rates $w\left(\mathbf{s}_{l} \rightarrow \mathbf{s}_{l l}^{\prime}\right), l=1$, $\ldots, n=N * M$.

(3) Calculate the total rate $w_{T}=\sum_{l} w\left(\mathbf{s}_{l} \rightarrow \mathbf{s}_{l}^{\prime}\right)$.

(4) Normalize all the transition rates as $v_{l}=w\left(\mathbf{s}_{l} \rightarrow \mathbf{s}_{l}^{\prime}\right) /$ $w_{T}$.

(5) Build the cumulative distribution $F\left(v_{l}\right)$ from the probability mass function $v_{l}$.

(6) Calculate the time $\Delta t$ to the next opinion flip by drawing from an exponential distribution with mean $1 / w_{T}$, that is, chooses a real number $0 \leq r \leq 1$ from a uniform distribution and sets $\Delta t=-w_{T}^{-1} \log (r)$.
(7) Identify the lth opinion that flips from $s_{l}$ to $-s_{l}$, by drawing from a discrete distribution with probability $v_{l}=w\left(s_{l}\right) / w_{T}$, that is, draws a real random number $0 \leq s \leq 1$ from a uniform distribution and chooses $l$ so that $F\left(v_{l-1}\right)<s<F\left(v_{l}\right)$.

(8) Update the state vector and return to step 2 or quit.

We stress note that the time step $\Delta t$ is not fixed and is roughly inversely proportional to the total rate $w_{T}=\sum_{l} w$ $\left(\mathbf{s}_{l} \rightarrow \mathbf{s}_{l}^{\prime}\right)$, and therefore, it depends on the number of individuals in the group, on the network structure, and its endogenous properties.

\section{Conflicts of Interest}

The authors declare that there is no actual or potential conflict of interest in relation to this article.

\section{References}

[1] J. Barnett, "Adapting to climate change in pacific island countries: the problem of uncertainty," World Development, vol. 29, no. 6, pp. 977-993, 2001.

[2] T. J. Vogus and K. M. Sutcliffe, "Organizational resilience: towards a theory and research agenda," in 2007 IEEE International Conference on Systems, Man and Cybernetics, pp. 34183422, Montreal, QC, Canada, October 2007.

[3] M. K. Linnenluecke, A. Griffiths, and M. Winn, "Extreme weather events and the critical importance of anticipatory adaptation and organizational resilience in responding to impacts," Business Strategy and the Environment, vol. 21, no. 1, pp. 17-32, 2012.

[4] G. S. van der Vegt, P. Essens, M. Wahlström, and G. George, "Managing risk and resilience," Academy of Management Journal, vol. 58, no. 4, pp. 971-980, 2015.

[5] G. Hamel and L. Välikangas, "The quest for resilience," Harvard Business Review, vol. 81, no. 9, pp. 52-63, 2003.

[6] R. Bhamra, S. Dani, and K. Burnard, "Resilience: the concept, a literature review and future directions," International Journal of Production Research, vol. 49, no. 18, pp. 5375-5393, 2011.

[7] K. M. Sutcliffe and T. J. Vogus, "Organizing for resilience," in Positive Organizational Scholarship: Foundations of a New Discipline, K. S. Cameron, J. E. Dutton, and R. E. Quinn, Eds., pp. 94-110, Berrett Koeller, San Francisco, CA, USA, 2003.

[8] C. S. Holling, "Resilience and stability of ecological systems," Annual Review of Ecology and Systematics, vol. 4, no. 1, pp. 1-23, 1973.

[9] G. S. Cumming, G. Barnes, S. Perz et al., "An exploratory framework for the empirical measurement of resilience," Ecosystems, vol. 8, no. 8, pp. 975-987, 2005.

[10] B. Walker, C. S. Holling, S. R. Carpenter, and A. P. Kinzig, "Resilience, adaptability and transformability in socialecological systems," Ecology and Society, vol. 9, no. 2, 2004.

[11] Y. Sheffi and J. B. Rice Jr., "A supply chain view of the resilient enterprise," MIT Sloan Management Review, vol. 47, no. 1, pp. 41-48, 2005.

[12] L. Riolli and V. Savicki, "Information system organizational resilience,” Omega, vol. 31, no. 3, pp. 227-233, 2003. 
[13] A. Rose, "Defining and measuring economic resilience to disasters," Disaster Prevention and Management: An International Journal, vol. 13, no. 4, pp. 307-314, 2004.

[14] A. Annarelli and F. Nonino, "Strategic and operational management of organizational resilience: current state of research and future directions," Omega, vol. 62, pp. 1-18, 2016.

[15] C. A. Lengnick-Hall, T. E. Beck, and M. L. Lengnick-Hall, "Developing a capacity for organizational resilience through strategic human resource management," Human Resource Management Review, vol. 21, no. 3, pp. 243-255, 2011.

[16] E. A. Mamouni Limnios, T. Mazzarol, A. Ghadouani, and S. G. M. Schilizzi, "The resilience architecture framework: four organizational archetypes," European Management Journal, vol. 32, no. 1, pp. 104-116, 2014.

[17] J. McCray, A. Palmer, and N. Chmiel, "Building resilience in health and social care teams," Personnel Review, vol. 45, no. 6, pp. 1132-1155, 2016.

[18] P. B. C. Morgan, D. Fletcher, and M. Sarkar, "Defining and characterizing team resilience in elite sport," Psychology of Sport and Exercise, vol. 14, no. 4, pp. 549-559, 2013.

[19] G. Pumpuni-Lenss, T. Blackburn, and A. Garstenauer, "Resilience in complex systems: an agent-based approach," Systems Engineering, vol. 20, no. 2, pp. 158-172, 2017.

[20] S. H. Stroeve and M. H. C. Everdij, "Agent-based modelling and mental simulation for resilience engineering in air transport," Safety Science, vol. 93, pp. 29-49, 2017.

[21] P. P. Datta, "A complex system, agent based model for studying and improving the resilience of production and distribution networks," 2007, http://hdl.handle.net/1826/1757.

[22] W. N. Adger, "Social capital, collective action, and adaptation to climate change," Economic Geography, vol. 79, no. 4, pp. 387-404, 2003.

[23] J. P. Stephens, E. D. Heaphy, A. Carmeli, G. M. Spreitzer, and J. E. Dutton, "Relationship quality and virtuousness: emotional carrying capacity as a source of individual and team resilience," The Journal of Applied Behavioral Science, vol. 49, no. 1, pp. 13-41, 2013.

[24] G. Carbone and I. Giannoccaro, "Model of human collective decision-making in complex environments," The European Physical Journal B, vol. 88, no. 12, p. 339, 2015.

[25] I. De Vincenzo, I. Giannoccaro, G. Carbone, and P. Grigolini, "Criticality triggers the emergence of collective intelligence in groups,” Physical Review E, vol. 96, no. 2, 2017.

[26] S. D. Dionne, H. Sayama, C. Hao, and B. J. Bush, "The role of leadership in shared mental model convergence and team performance improvement: an agent-based computational model," The Leadership Quarterly, vol. 21, no. 6, pp. 10351049, 2010.

[27] K. A. McHugh, F. J. Yammarino, S. D. Dionne, A. Serban, H. Sayama, and S. Chatterjee, "Collective decision making, leadership, and collective intelligence: tests with agent-based simulations and a field study," The Leadership Quarterly, vol. 27, no. 2, pp. 218-241, 2016.

[28] S. Kauffman and S. Levin, "Towards a general theory of adaptive walks on rugged landscapes," Journal of Theoretical Biology, vol. 128, no. 1, pp. 11-45, 1987.

[29] S. A. Kauffman and E. D. Weinberger, "The NK model of rugged fitness landscapes and its application to maturation of the immune response," Journal of Theoretical Biology, vol. 141, no. 2, pp. 211-245, 1989.
[30] J. W. Rivkin, "Reproducing knowledge: replication without imitation at moderate complexity," Organization Science, vol. 12, no. 3, pp. 274-293, 2001.

[31] J. W. Rivkin and N. Siggelkow, "Balancing search and stability: interdependencies among elements of organizational design," Management Science, vol. 49, no. 3, pp. 290-311, 2003.

[32] R. J. Glauber, "Time-dependent statistics of the Ising model," Journal of Mathematical Physics, vol. 4, no. 2, pp. 294-307, 1963.

[33] N. Siggelkow and J. W. Rivkin, "Speed and search: designing organizations for turbulence and complexity," Organization Science, vol. 16, no. 2, pp. 101-122, 2005.

[34] T. Khanna and J. W. Rivkin, "Estimating the performance effects of business groups in emerging markets," Strategic Management Journal, vol. 22, no. 1, pp. 45-74, 2001.

[35] N. Siggelkow and D. A. Levinthal, "Temporarily divide to conquer: centralized, decentralized, and reintegrated organizational approaches to exploration and adaptation," Organization Science, vol. 14, no. 6, pp. 650-669, 2003.

[36] S. K. Ethiraj and D. Levinthal, "Modularity and innovation in complex systems," Management Science, vol. 50, no. 2, pp. 159-173, 2004.

[37] I. Giannoccaro, "Assessing the influence of the organization in the supply chain management using NK simulation," International Journal of Production Economics, vol. 131, no. 1, pp. 263-272, 2011.

[38] I. Giannoccaro, "Adaptive supply chains in industrial districts: a complexity science approach focused on learning," International Journal of Production Economics, vol. 170, pp. 576589, 2015.

[39] A. Capaldo and I. Giannoccaro, "How does trust affect performance in the supply chain? The moderating role of interdependence," International Journal of Production Economics, vol. 166, pp. 36-49, 2015.

[40] A. Capaldo and I. Giannoccaro, "Interdependence and network-level trust in supply chain networks: a computational study," Industrial Marketing Management, vol. 44, pp. 180195, 2015.

[41] I. Giannoccaro, A. Nair, and T. Choi, "The Impact of control and complexity on supply network performance: an empirically informed investigation using NK simulation analysis," Decision Sciences, 2017.

[42] P. Agarwal, M. Sahai, V. Mishra, M. Bag, and V. Singh, "A review of multi-criteria decision making techniques for supplier evaluation and selection," International Journal of Industrial Engineering Computations, vol. 2, no. 4, pp. 801810, 2011.

[43] N. Siggelkow, "Firms as systems of interdependent choices," Journal of Management Studies, vol. 48, no. 5, pp. 11261140, 2011.

[44] C. M. Bordogna and E. V. Albano, "Dynamic behavior of a social model for opinion formation," Physical Review E, vol. 76, no. 6, 2007.

[45] C. M. Bordogna and E. V. Albano, "Statistical methods applied to the study of opinion formation models: a brief overview and results of a numerical study of a model based on the social impact theory," Journal of Physics: Condensed Matter, vol. 19, no. 6, 2007.

[46] D. Sornette, "Physics and financial economics (1776-2014): puzzles, Ising and agent-based models," Reports on Progress in Physics, vol. 77, no. 6, 2014. 
[47] I. Giannoccaro and G. Carbone, "An Ising-based dynamic model to study the effect of social interactions on firm absorptive capacity," International Journal of Production Economics, vol. 194, pp. 214-227, 2017.

[48] D. Stauffer, "Social applications of two-dimensional Ising models," American Journal of Physics, vol. 76, no. 4, pp. 470473, 2008.

[49] C. M. Bordogna and E. V. Albano, "Phase transitions in a model for social learning via the internet," International Journal of Modern Physics C, vol. 12, no. 8, pp. 12411250, 2001.

[50] W. X. Zhou and D. Sornette, "Self-organizing Ising model of financial markets," The European Physical Journal B, vol. 55, no. 2, pp. 175-181, 2007.

[51] H. B. Stauffer, "Application of Bayesian statistical inference and decision theory to a fundamental problem in natural resource science: the adaptive management of an endangered species," Natural Resource Modeling, vol. 21, no. 2, pp. 264284, 2008.

[52] S. Y. Ponomarov and M. C. Holcomb, "Understanding the concept of supply chain resilience," The International Journal of Logistics Management, vol. 20, no. 1, pp. 124-143, 2009.

[53] A. Rose, "Economic resilience to natural and man-made disasters: multidisciplinary origins and contextual dimensions," Environmental Hazards, vol. 7, no. 4, pp. 383-398, 2007.

[54] H. Carvalho, A. P. Barroso, V. H. Machado, S. Azevedo, and V. Cruz-Machado, "Supply chain redesign for resilience using simulation," Computers \& Industrial Engineering, vol. 62, no. 1, pp. 329-341, 2012.

[55] K. E. Weick, "Enacted sensemaking in crisis situations," Journal of Management Studies, vol. 25, no. 4, pp. 305-317, 1988.

[56] B. Smit and J. Wandel, "Adaptation, adaptive capacity and vulnerability," Global Environmental Change, vol. 16, no. 3, pp. 282-292, 2006.

[57] M. Bruneau, S. E. Chang, R. T. Eguchi et al., "A framework to quantitatively assess and enhance the seismic resilience of communities," Earthquake Spectra, vol. 19, no. 4, pp. 733$752,2003$.

[58] F. Luthans, C. M. Youssef, and B. J. Avolio, Psychological Capital: Developing the Human Competitive Edge, Oxford University Press, Oxford, UK, 2007.

[59] E. E. Werner and R. S. Smith, "Journeys from childhood to midlife. Risk, resilience and recover," Social Anthropology, vol. 13, no. 2, pp. 245-246, 2001.

[60] A. S. Masten, "Ordinary magic: resilience processes in development," American Psychologist, vol. 56, no. 3, pp. 227-238, 2001.

[61] G. M. Fisk and A. M. Dionisi, "Chapter 7 building and sustaining resilience in organizational settings: the critical role of emotion regulation," in Research on Emotion in Organizations, pp. 167-188, 2010.

[62] D. L. Coutu, "How resilience works," Harvard Business Review, vol. 80, no. 5, pp. 46-50, 2002.

[63] J. B. Bennett, C. A. Aden, K. Broome, K. Mitchell, and W. D. Rigdon, "Team resilience for young restaurant workers: research-to-practice adaptation and assessment," Journal of Occupational Health Psychology, vol. 15, no. 3, pp. 223-236, 2010.

[64] D. P. Ashmos and G. P. Huber, "The systems paradigm in organization theory: correcting the record and suggesting the future," Academy of Management Review, vol. 12, no. 4, pp. 607-621, 1987.

[65] G. M. Alliger, C. P. Cerasoli, S. I. Tannenbaum, and W. B. Vessey, "Team resilience: how teams flourish under pressure," Organizational Dynamics, vol. 44, no. 3, pp. 176-184, 2015.

[66] P. R. Lawrence and J. W. Lorsch, "Differentiation and integration in complex organizations," Administrative Science Quarterly, vol. 12, no. 1, pp. 1-47, 1967.

[67] M. B. Takeda and M. M. Helms, “"Bureaucracy, meet catastrophe": analysis of the tsunami disaster relief efforts and their implications for global emergency governance," International Journal of Public Sector Management, vol. 19, no. 2, pp. 204217, 2006.

[68] E. D. Weinberger, "Santa Fe Institute working paper: 1996-02-003,” 1996, https://www.santafe.edu/research/results/ working-papers/np-completeness-of-kauffmans-n-k-modela-tuneably-.

[69] J. W. Rivkin, "Imitation of complex strategies," Management Science, vol. 46, no. 6, pp. 824-844, 2000.

[70] P. J. DiMaggio and W. W. Powell, “The iron cage revisited: institutional isomorphism and collective rationality in organizational fields," American Sociological Review, vol. 48, no. 2, p. 147, 1983.

[71] W. Weidlich, "Physics and social science - the approach of synergetics," Physics Reports, vol. 204, no. 1, pp. 1-163, 1991.

[72] D. T. Gillespie, "A general method for numerically simulating the stochastic time evolution of coupled chemical reactions," Journal of Computational Physics, vol. 22, no. 4, pp. 403-434, 1976.

[73] D. T. Gillespie, "Exact stochastic simulation of coupled chemical reactions," The Journal of Physical Chemistry, vol. 81, no. 25, pp. 2340-2361, 1977.

[74] D. Barkoczi and M. Galesic, "Social learning strategies modify the effect of network structure on group performance," Nature Communications, vol. 7, article 13109, 2016.

[75] R. Hastie and T. Kameda, "The robust beauty of majority rules in group decisions," Psychological Review, vol. 112, no. 2, pp. $494-508,2005$. 


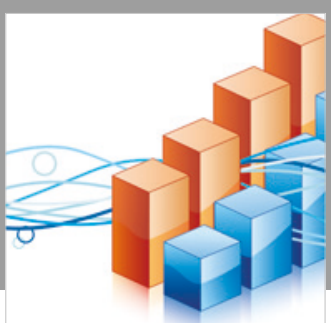

Advances in

Operations Research

\section{-n-m}
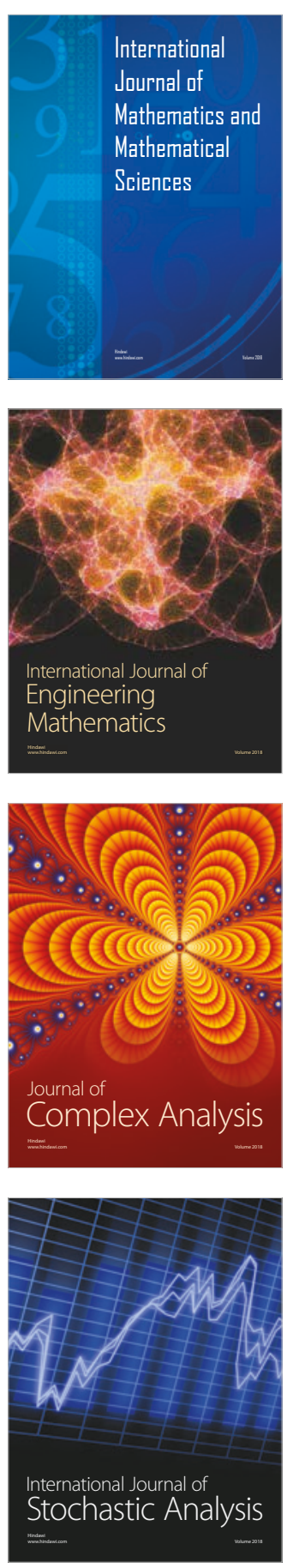
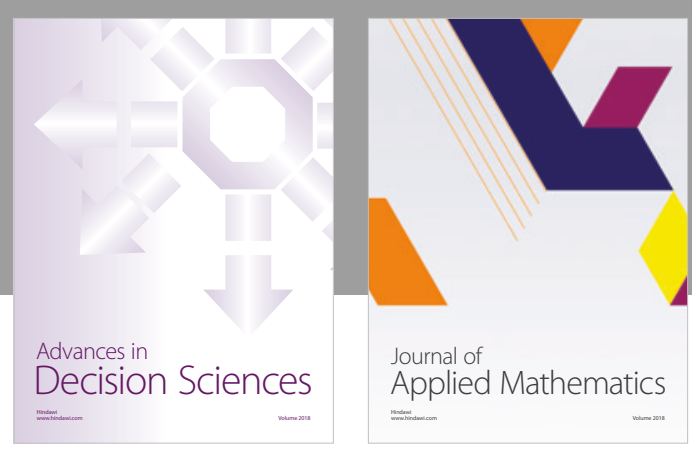

Journal of

Applied Mathematics
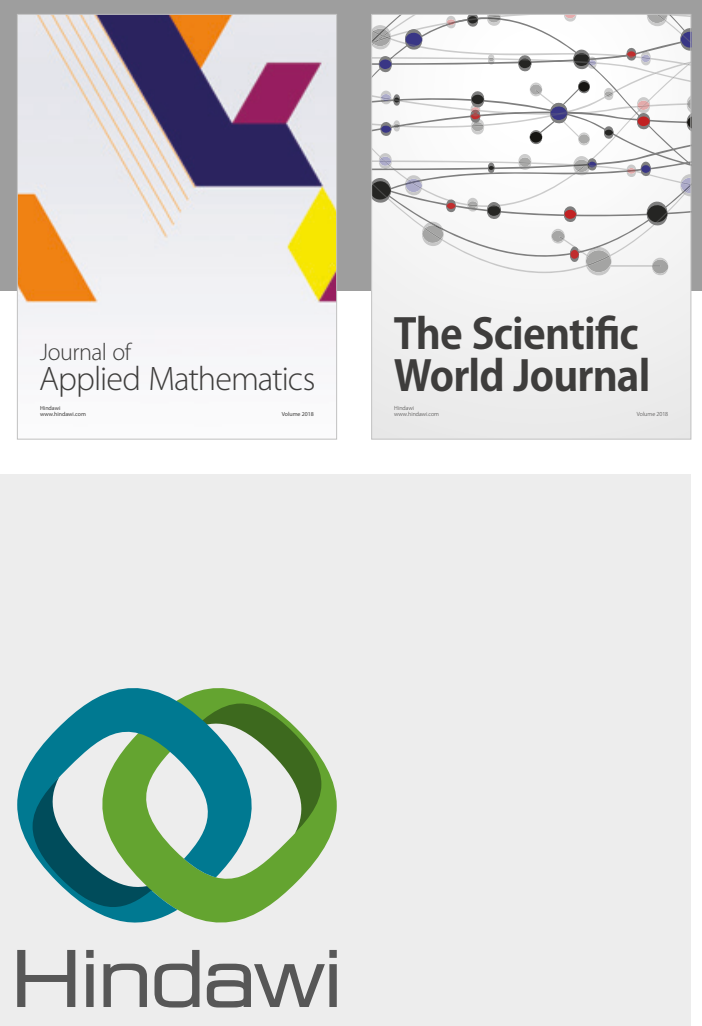

Submit your manuscripts at

www.hindawi.com

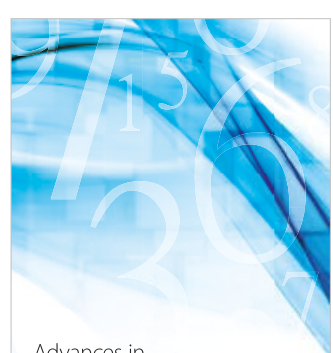

Advances in
Numerical Analysis
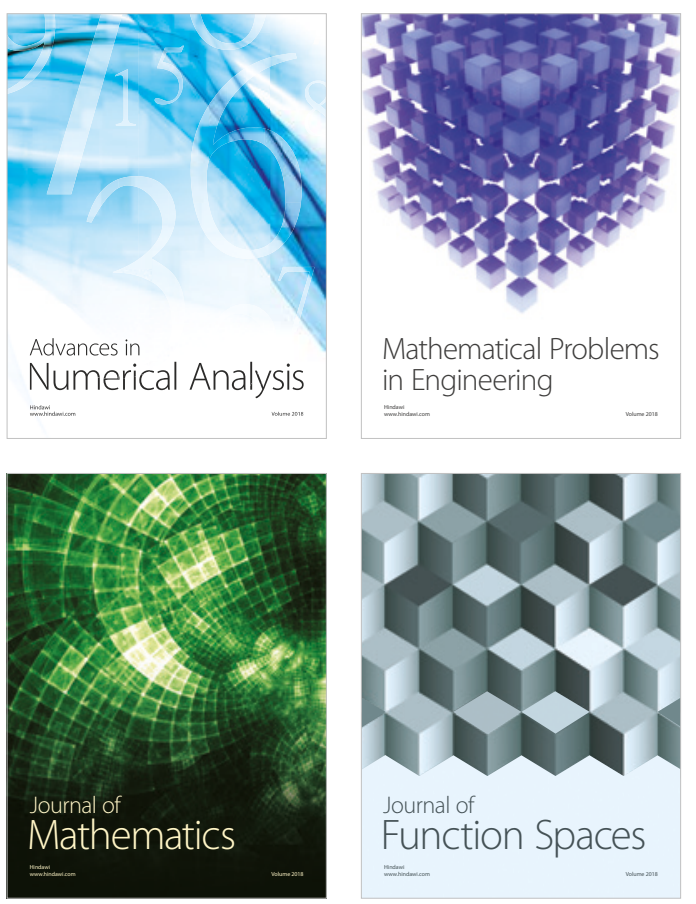

Mathematical Problems in Engineering

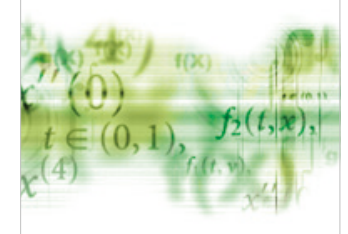

International Journal of

Differential Equations

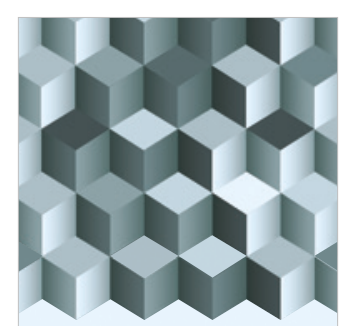

Journal of

Function Spaces
The Scientific

World Journal

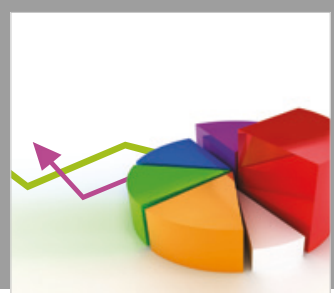

Journal of

Probability and Statistics
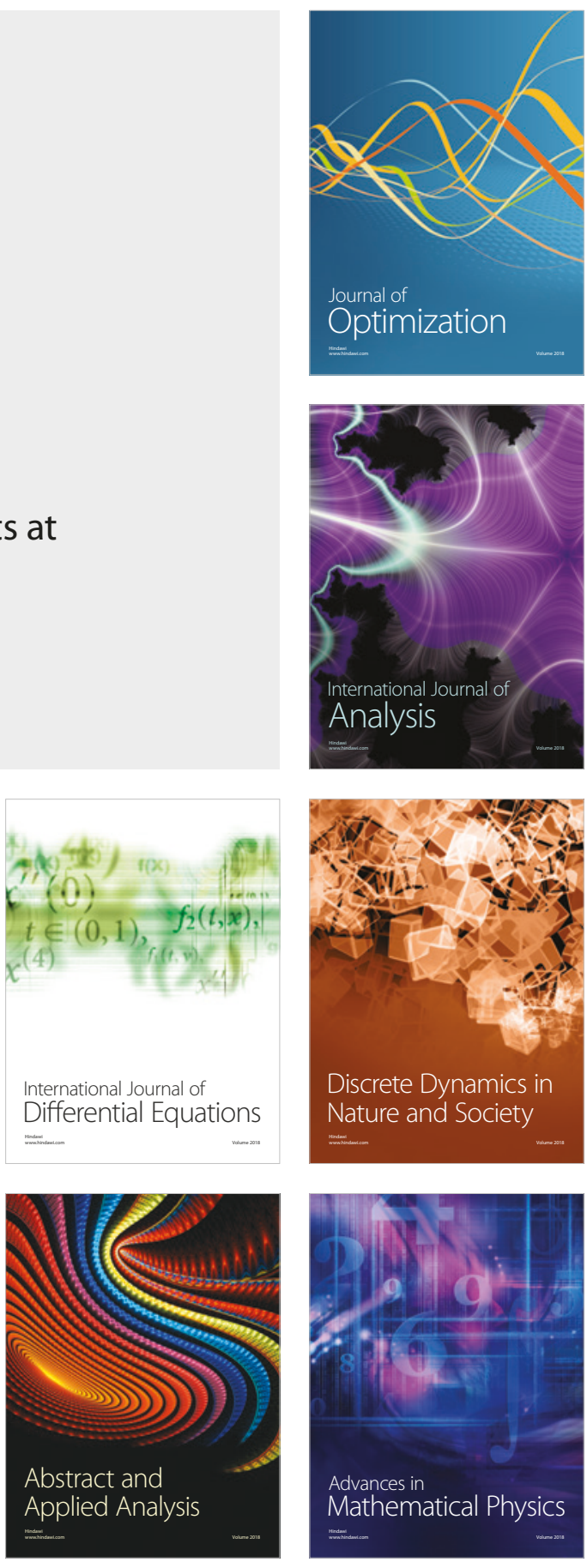